\title{
A Fast Fractal Model Based ECG Compression Technique
}

\author{
Lin Yin ${ }^{1, a}$, Fang $Y u^{\star^{1, b}}$ \\ ${ }^{1}$ Electronicsand information engineering college, Tongji University, ShangHai, China \\ aemail: findingsea@163.com, bemail: fangyu@tongji.edu.cn
}

Keywords: Fractal; Dynamic Comparison Sequence

\begin{abstract}
ECG monitoring equipmentwill generate a huge mount of ECG signals, which causes enormous pressure on server storage and transmission. Therefore, many compression techniques are proposed to overcome this problem including some fractal based lossy ECG compression techniques. However, basic fractal based techniques cannot be used widely on some large-scale applications because of its high computation complexity.In this paper, an improved faster fractal model is proposed to solve this problem. The most important and time-cost step in the basic fractal model is the comparison of range and domain blocks. Traditionally, the comparison sequence is fixed and static. In the new improved fractal model, the sequence is changed to be dynamic toaccelerate the compression. It is found that the improved fractal based technique can achieve same compression ratio and similar Percentage Residual Difference(PRD) error with 4 times faster than the basic fractal basedtechnique.
\end{abstract}

\section{Introduction}

The amount of ECG signal generated by kinds ofmonitor equipmentis huge, so it brings tremendous pressure on data storage to hospital and data storage service providers. Meanwhile, many e-health applications and healthcare organizations require access to these records, soit brings pressure on transmission. Especially, mobile healthcare is increasing to a great extent around global in recently years and itexacerbates these two problems undoubtedly.

In order to reduce the pressure on storage and transmission, many ECG compression techniques have been created and implemented. Generally, these techniques can be divided into two main categories: lossless and lossy compression. Losslesstechniques can keep the full structure of origin ECG signal without losing any information, but its compression ratio is low and cannot satisfy the requirement of large applications. On the other hand, although lossy technique just reconstruct approximated version of the origin ECG signal, the differences between the origin ECG signal and the reconstructed ECG signal are very small, so hospitals and healthcare sectors still can use the reconstructed signal on medical research and diagnosis. More importantly, with lossy compression technique, it is possible to achieve higher compression ratio. There are two major types in lossy compression: time domain compression techniques [1-3] and transform domain techniques [4-7]. Wavelet-based technique [4], real-time ECG compression and transmission technique [5], 1D to 2D compression technique [6] and an ECG compression using Jpeg2000 algorithm [7]are all wellknown and excellent ECG compression techniques. But in the term of compression ratio, they can'tcompare with fractal based technique.Thesetechniques mentioned above can achieve 10 to 30 compression ratio while fractal based technique can achieve more than 40 compression ratio [89].Most of existing compression techniques no matter time domain or transform domainare based on R peak detection or other ECG parameter detection [9]. Therefore, they can not provide high compression ratio performance like fractal based techniques since the fractal model has a huge advantage in dealing with such signal with self-similarity characteristic.It has been proved that the periodic self-similarity characteristic of the ECG signal can be a powerful feature to be used in fractal based compression techniques [9]. The proposed technique in this paper is based on fractal model.

At present, the biggest problem in fractal based techniquesis effectiveness. Although using fractal model can achieve higher compression ratio, the process of compression is so complicated 
that whole algorithm requires a lot of time to execute. It is the main reason that fractal based technique haven't been used in industry-level applications widely now. The major goal of this papers is to reduce the execution time of the basic fractal based technique.The most time-cost part of fractal based techniqueis the comparison of range and domain blocks. In the comparison process of fractal model, each range block needs to compare with each domain block to find the most similarone. Finally, the indexes of the most similar domain block and some related parameters are stored into the compressed file. In order to improve the fractal based technique, dynamic comparison sequence is proposed in this paper to reduce the number of comparison of range and domain blocks. Dynamic comparison sequencemakes each range block to find the domain that meets the requirement without comparing whole domain blocks. The fractal model using dynamic domain sequence is implemented in this paper and evaluatedby three important measures: compression ratio, signal reconstruction error and compression execution time. Experiment results shows that the proposed improved fractal based technique can achieve same compression ratio and similar reconstruction error with only $1 / 4$ compression time of the basic fractal based technique.

\section{Related work}

Many researchers have conducted investigation on fractal based ECG compressiontechniques. A. Khalaj and H. Miar Naimi proposed new efficient fractal based compression method for electrocardiogram signals[8]. Their method is based on the fractal model and IFS(Iterated Function System). In their model the origin ECG signal is divided into non-overlapped blocks called range and the down-sampled copy version is divided into overlapped blocks called domain. For each range block, their method find the most similar domain then generates the fractal transformation parameters. The final compressed file store the indexes of range and domain blocks and the parameters. The indexes and parameters are used to reconstruction the ECG signal.

Ayman Ibaida, Dhiah Al-Shammary and Ibrahim Khalil proposed a cloud enable fractal based ECG compression technique[9]. Their work is somewhat similar to A. Khalaj and H. Miar Naimi's, in their method they used two transformation parameters when in A. Khalaj and H. Miar Naimi's only one parameter is used. Their technique can achieve high compression ratio with low reconstruction error.

According to Ayman Ibaida's paper [9], The self-similarity characteristic of ECG signal is powerful feature that can be used in fractal model.The improved fractal model proposed in this paper is based on their model and can achieve same compression ratio and similar reconstruction error with less compression time. The evaluation section shows the compression performance comparison.

The basic coefficients used to describe the transformation relationship are scale (S) and offset (O).The scale coefficient is defined as the greatest difference among ECG instances in the range block divided by the greatest difference among the ECG instances in the domain block. Eq. (1) shows the calculation of scale coefficient[10].

$\mathrm{S}=\frac{\mathrm{n} \sum_{\mathrm{i}=1}^{\mathrm{n}} \mathrm{D}(\mathrm{i}) \mathrm{R}(\mathrm{i})-\sum_{\mathrm{i}=1}^{\mathrm{n}} \mathrm{R}(\mathrm{i}) \sum_{\mathrm{i}=1}^{\mathrm{n}} \mathrm{D}(\mathrm{i})}{\mathrm{n} \sum_{\mathrm{i}=1}^{\mathrm{n}} \mathrm{D}(\mathrm{i})^{2}-\left(\sum_{\mathrm{i}=1}^{\mathrm{n}} \mathrm{D}(\mathrm{i})\right)^{2}}$

The offset coefficient is defined as the difference between the average ECG instance value in the range block and the average ECG instance value in the domain block.Eq. (2) shows the calculation of offset coefficient[10].

$\mathrm{O}=\frac{1}{\mathrm{n}}\left(\sum_{\mathrm{i}=1}^{\mathrm{n}} \mathrm{R}(\mathrm{i})-\mathrm{S} \sum_{\mathrm{i}=1}^{\mathrm{n}} \mathrm{D}(\mathrm{i})\right)$

Eqs. (3) shows how to use scale and offset coefficients and domain block to generate the range block[10].

$\mathrm{R} \approx \mathrm{S} \times \mathrm{D}+\mathrm{O}$

These parameters are calculated for each range block and domain blockwhich need to be compared and describe the transformation relationship between range and domain block. 
The basic fractal model proposed in Ayman Ibaida's paper uses static comparison sequence, which means each range block needs to compare with all domain blocks and calculate the fractal coefficients for all domain blocks. The comparison process is shown in Fig. 1.

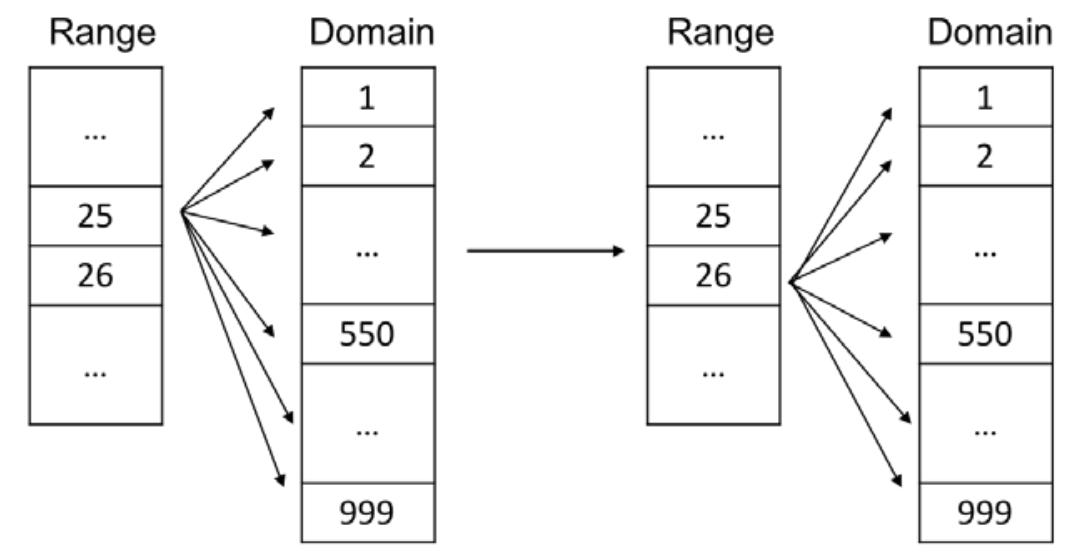

Fig 1. The comparison process using static sequence.

In ECG signal, two adjacent range blocks are similar on the value of some parameters. So when one domain block is similar to a range block greatly, it has a high possibility to be similar to the next range block. But this feature is not used in the fractal model using static comparison sequence which is shown in Fig. 2. These domain blocks in the black boxes are more similar to the current range block obviously, but the fractal model using static comparison sequence doesn't care about this feature and it still compares the range block with all domain blocks one by one.

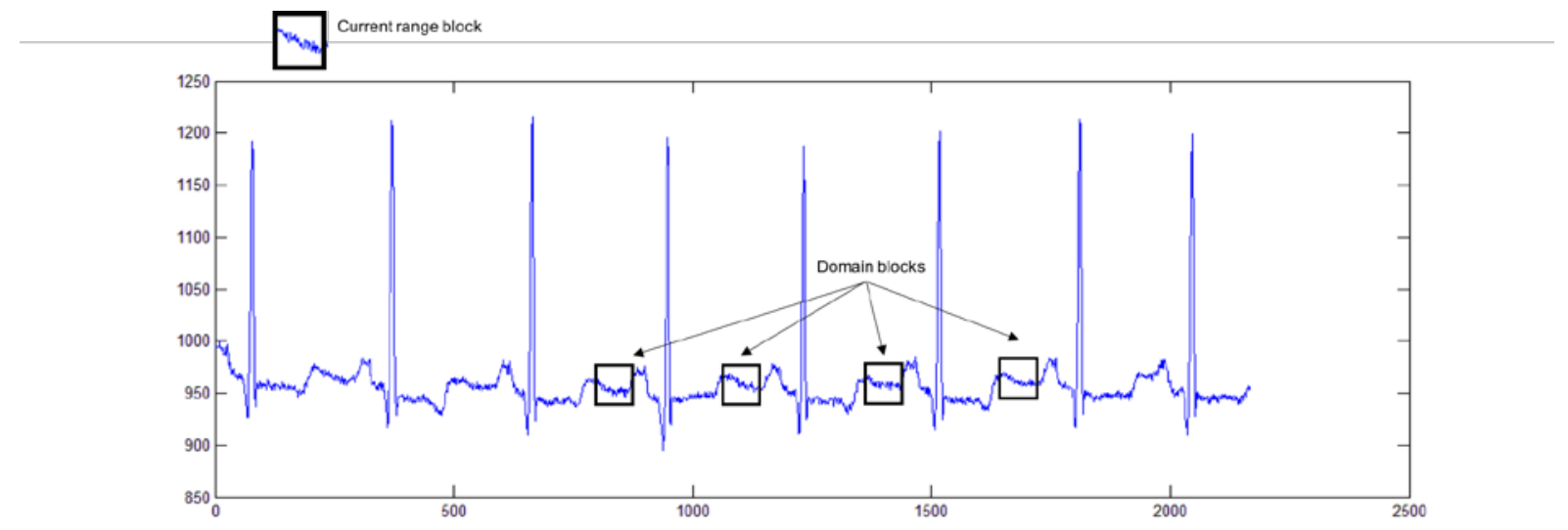

Fig 2. The defect of static comparison sequence.

\section{Compression algorithm}

In our improved fractal model, dynamic comparison sequence is used to reduce the number of comparison and accelerate the comparison process. The comparison sequence of current range block is generated by the comparison result of previous range block. It can always keep the more similar domain blocks in the front of the comparison sequence for each range block and find the domain block meeting the requirement to break the iteration without comparing with whole domain. The comparison process is shown in Fig. 3. 


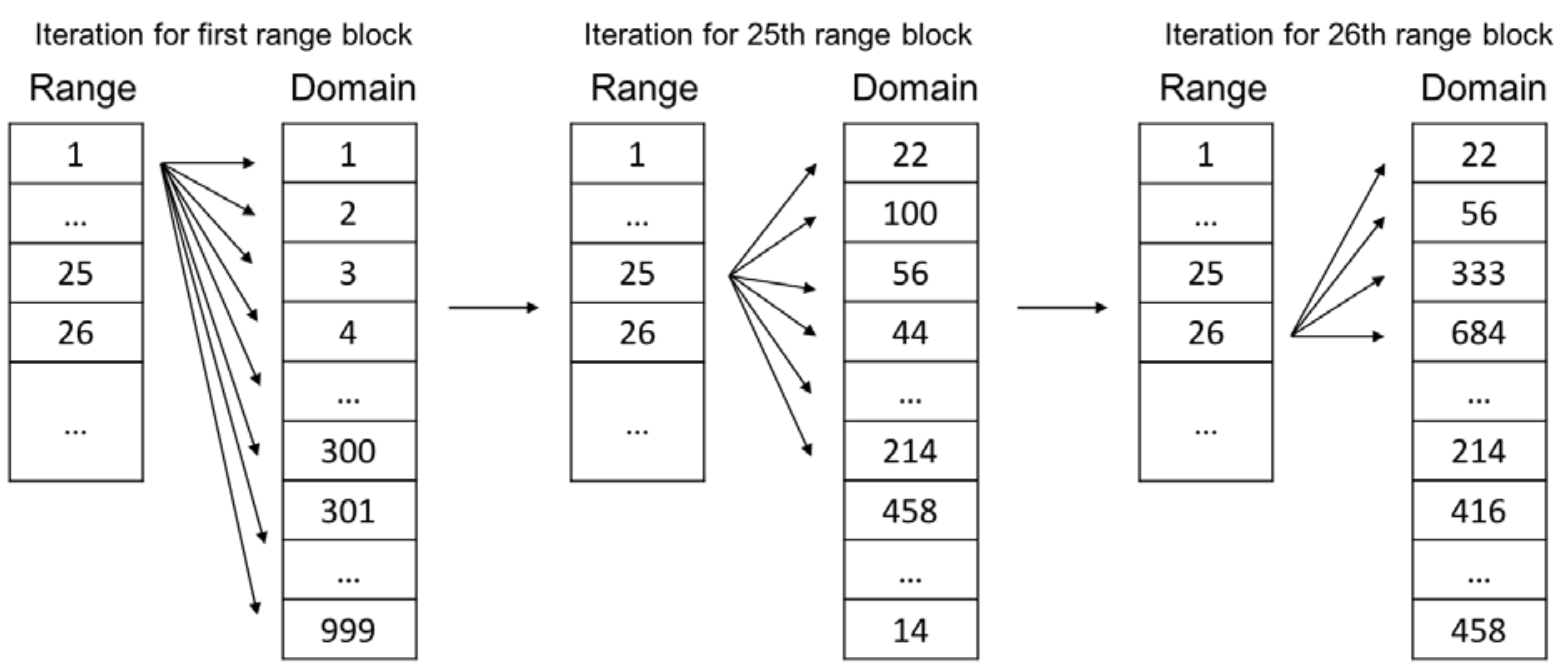

Fig 3. The comparison process using dynamic sequence.

In the proposed improved fractal based compression technique, for each range block algorithm finds the domain block with high similarity. There is a queue storing the indexes of domain block. For each given range block, the algorithm iterates through domain blocks according the index order stored in the queue and calculates the fractal coefficients. When the current domain block is similar to the given range block greatly, algorithm keeps the corresponding index in place. When the current domain block is not similar to the given range block, algorithm moves the corresponding index to the tail of the queue. After found the domain block meeting requirement, the indexes queue has been adjusted and can be used as the comparison sequenceof the next range block. Fractal Root Mean Square (RMS) is used as the similarity measurement between range block and domain block.Eq. (4) shows the calculation of the RMS[9].

$$
\begin{aligned}
R M S=\sqrt{\frac{1}{n}\left[\sum_{i=1}^{n} R(i)^{2}+S\left(S \sum_{i=1}^{n} D(i)^{2}-2 \sum_{i=1}^{n} R(i) D(i)+2 O \sum_{i=1}^{n} D(i)\right)\right.} \\
\left.+O\left(n O-2 \sum_{i=1}^{n} R(i)\right)\right]
\end{aligned}
$$

If the current RMS is less than the previous stored value, algorithm will store the current calculated fractal coefficient in the output parameters and update the RMS value. If the current RMS is less than some threshold, algorithm will break the current iteration in advance and store the corresponding fractal coefficients to the compressed file.

In order to further improve the performance, some coding techniques can be used.Some arrays can be calculated before the iteration and stored in the memory so that program can run faster with a little more memory usage. Meanwhile, affine transforms [11] are applied to help to achieve higher similarity between the range and domain blocks. Four different kinds of affine transformations are used in the algorithm and the affine transformation encoding number will be store in the final compressed file.

The final compressed file contains several rows, each row includes the fractal coefficient (scale and offset), the domain block index and the affine transformation encoding number.

Compression Algorithm

\section{INPUTS:}

$\mathrm{R}$ represents the origin ECG signal

bs represents the block size

js represents the jump step of the search loop in domain

OUTPUTS:

so represents the scale value for the selected domain block

oo represents the offset value for the selected domain block

io represents the index of the selected domain block 
aff0 represents the affine transformation encoding value for the selected domain block

// parameter initial

$\mathrm{D}=$ the down-sampled version of origin ECG signal

numOfRBlocks=the number of range blocks

numOfDBlocks $=$ the number of domain blocks

queue $=$ one-dimensional queue, queue[i] represents the index of domain block

$q=0$, represents index of queue

// queue initial

while $(\mathrm{q}<$ numOfDBlocks) queue.add $(\mathrm{q}++)$

for each range block $\mathrm{Ri}$ in $\mathrm{R}$ do:

sumr $=\operatorname{sum}(\mathrm{Ri})$

sumr2=sum(Ri.$\wedge 2)$

$\mathrm{rms} 0=1000$

$\mathrm{SO}=0$

$\mathrm{OO}=0$

io $=0$

aff $0=0$

$\mathrm{n}=\mathrm{bs}$

toAppendList=one-dimensional queue storing the element reomved from queue

for each element q in queue do:

$\mathrm{j}=$ queue $[\mathrm{q}]$

for $\mathrm{a}=0$ to 1 do:

$\mathrm{Dj}=\mathrm{jth}$ domain block

$\operatorname{sumrd}=\operatorname{sum}(\mathrm{Ri} * \mathrm{Dj})$

sumd $=\operatorname{sum}(\mathrm{Dj})$

$\operatorname{sumd} 2=\operatorname{sum}(\mathrm{Dj} . \wedge 2)$

$\mathrm{s}=((\mathrm{n} *$ sumrd $)-($ sumr $*$ sumd $[\mathrm{j}])) /(\mathrm{n} *$ sumd2 $[\mathrm{j}]-\operatorname{sumd}[\mathrm{j}] \wedge 2)$

$\mathrm{s}=($ sumr $-\mathrm{s} *$ sumd[j]) / n

$\mathrm{rms}=\operatorname{sqrt}((\operatorname{sumr} 2+\mathrm{s} *(\mathrm{~s} * \operatorname{sumd} 2[\mathrm{j}]-2 * \operatorname{sumrd}+2 * \mathrm{o} * \operatorname{sumd}[\mathrm{j}])+\mathrm{o} *(\mathrm{n} * \mathrm{o}-2 * \operatorname{sumr}))$

/ n)

if $\mathrm{rms}<\mathrm{rms} 0$ :

$$
\begin{aligned}
& \text { so }=\mathrm{s} \\
& \text { oo }=\mathrm{o} \\
& \text { io }=\mathrm{j} \\
& \text { aff0 }=\mathrm{a} \\
& \text { rms0 }=\mathrm{rms}
\end{aligned}
$$

end if

end for

if rms $0<5.75$ do: break

end if

if $\mathrm{rms} 0>15$ do:

remove $q$ from queue

add $q$ to toAppendList

end if

end for

add toAppendList to queue

write so, oo, io, aff0 to compressd file

end for 


\section{Evaluation}

Three measurements are used in this paper to measure theperformance of the proposed improved fractal model and compare it with the basic fractal model.The compression ratio (CR) is a very important measurement that reflects the ratio between the original signal and the compressed signal. Eq. (5) shows the calculation of the compression ratio.

$$
C R=\frac{\text { the size of original file }}{\text { the size of compressed file }}
$$

The percentage residual difference (PRD) is an important measure that reflects the differences between the original signal and the reconstructed signal. Eq. (6) shows the calculation of the percentage residual difference.

$P R D=\sqrt{\frac{\sum_{i=1}^{N}\left(R_{i}-R_{r e i}\right)^{2}}{\sum_{i=1}^{N} R_{i}{ }^{2}}}$

$\mathrm{R}$ represents the original ECG signal and $\mathrm{R}_{\mathrm{re}}$ represents the reconstructed version.

The improved fractal model based algorithm is measured using the MIT-BIH Arrhythmia Database. Each ECG record is of 30 min length and collected from different patients. Due to the limitations of our equipment, the test for the improved fractal model in this paper just use 10 seconds of each record. If we use longer length data in the future work, the test result will be better (the value of PRD will be lower). However, even in the current situation, the improved fractal model still achieve good performance.

In the improved fractal mode,the block size used is 35 points and the jump step is 10 points.The model and the algorithm are implemented by Java language and experiments are executed on the retina MacBook Prowith Mac OS X system. The experiment result and the performance of the basic model and other ECG compression techniques are shown in Table 1. The proposed algorithm can achieve the compression ratio of 42 with the PRD value less than $2 \%$. In light of the fact that the similarity with the original data becomes meaningless when the PRD value is higher than 2\% [5]. Compared with the basic fractal model, the improved model can achieve same compression ratio and similar PRD error.

Table 1 CR and PRD for different compression techniques compared with ours.

\begin{tabular}{|c|c|c|c|c|c|c|c|c|c|c|c|c|}
\hline \multirow[t]{2}{*}{ Data } & \multicolumn{2}{|c|}{$\begin{array}{l}\begin{array}{l}\text { The improved } \\
\text { model }\end{array} \\
\end{array}$} & \multicolumn{2}{|c|}{ The basic model[9] } & \multicolumn{2}{|l|}{ [5] } & \multicolumn{2}{|l|}{$[6]$} & \multicolumn{2}{|l|}{ [7] } & \multicolumn{2}{|l|}{ [8] } \\
\hline & CR & PRD & CR & PRD & CR & PRD & CR & PRD & CR & PRD & CR & PRD \\
\hline 100 & 42 & 1.61 & 42 & 0.79 & 22.94 & 1.95 & 24 & 3.95 & 24 & 4.06 & 11.06 & 13.79 \\
\hline 101 & 42 & 1.40 & 42 & & & & & & & & & \\
\hline 102 & 42 & 1.03 & 42 & 1 & 25.9 & 1.39 & & & & & 10.17 & 14.2 \\
\hline 103 & 42 & 2.42 & 42 & 2.29 & 20.33 & 2.5 & & & & & 11 & 13.78 \\
\hline 104 & 42 & 1.14 & 42 & 0.96 & 22.94 & 1.67 & & & & & 11.2 & 13 \\
\hline 105 & 42 & 1.27 & 42 & 1.24 & 20.96 & 1.17 & & & & & 12 & 15.13 \\
\hline 106 & 42 & 1.97 & 42 & 1.65 & 19.55 & 1.77 & & & & & & \\
\hline 107 & 42 & 1.92 & 42 & 2.66 & 18.55 & 3.93 & & & & & & \\
\hline 108 & 42 & 0.75 & 42 & 0.53 & 23.11 & 0.77 & & & & & 8.22 & 15.42 \\
\hline 109 & 42 & 1.39 & 42 & 0.87 & 19.89 & 0.76 & & & & & 11 & 17.39 \\
\hline 111 & 42 & 1.01 & 42 & 0.92 & 22.99 & 1.03 & & & & & & \\
\hline 112 & 42 & 2.94 & 42 & 1.18 & 23.82 & 1 & & & & & & \\
\hline 113 & 42 & 2.87 & 42 & 2.4 & 19.96 & 2.89 & & & & & & \\
\hline 114 & 42 & 1.43 & 42 & 0.87 & 25.58 & 1.08 & & & & & & \\
\hline 117 & 42 & 1.63 & 42 & 1.45 & 24 & 1.17 & 24 & 1.72 & 13 & 1.18 & 4.5 & 14.64 \\
\hline 118 & 42 & 2.17 & 42 & & & & & & & & & \\
\hline 119 & 42 & 1.98 & 42 & 1.5 & 19 & 2.05 & 20 & 1.92 & 21 & 2.81 & & \\
\hline
\end{tabular}


The compression time comparison between the basic fractal model and the improved fractal model is shown in Table 2 and Table 3.The length of the ECG segment used in the experiment is 10 seconds. The unit is millisecond. In this paper, the basic model is implemented by ourselves according to the corresponding paper [9]. Both models use the block size of 35 and jump step of 10 and runon the same machine. As the tables show, the execution time of the improved model can be faster 4 times than the basic model.

Table 2 Execution time comparison of basic model and improved model

\begin{tabular}{|l|l|l|l|l|l|l|l|l|l|l|}
\hline Data & 100 & 101 & 102 & 103 & 104 & 105 & 106 & 107 & 108 & 109 \\
\hline The improved model & 199 & 136 & 127 & 94 & 135 & 102 & 116 & 289 & 171 & 186 \\
\hline The basic model & 539 & 433 & 417 & 400 & 408 & 406 & 401 & 555 & 597 & 579 \\
\hline
\end{tabular}

Table 3 Execution time comparison of basic model and improved model

\begin{tabular}{|l|l|l|l|l|l|l|l|l|l|}
\hline Data & 111 & 112 & 113 & 114 & 115 & 116 & 117 & 118 & 119 \\
\hline The improved model & 135 & 124 & 156 & 105 & 108 & 269 & 100 & 268 & 172 \\
\hline The basic model & 583 & 585 & 592 & 587 & 595 & 585 & 592 & 582 & 593 \\
\hline
\end{tabular}

\section{Conclusion}

In this paper, an improved faster fractal model based ECG compression technique is proposed to reduce the execution time of the basic model. The fractal model has been proved that can be used to be a good ECG compression technique with high compression ratio and low PRD error. However, the high computation complexity and long execution time makes the basic fractal model be difficult to used in large-scale applications. Dynamic sequence technique is used in this paper to optimize the comparison process of the range and domain blocks. Finally,the proposed faster fractal model can achieve same compression ratio and similar PRD error with only $1 / 4$ execution time of the basic model. It is possible to reduce the PRD error further which will be studied in the future work.

\section{References}

[1] Arnavut Z. ECG signal compression based on Burrows-Wheeler transformation and inversion ranks of linear prediction[J]. Biomedical Engineering, IEEE Transactions on, 2007, 54(3): 410-418.

[2] Fira C M, Goras L. An ECG signals compression method and its validation using NNs[J]. Biomedical Engineering, IEEE Transactions on, 2008, 55(4): 1319-1326.

[3] Huang B, Wang Y, Chen J. 2-D compression of ECG signals using ROI mask and conditional entropy coding[J]. IEEE transactions on bio-medical engineering, 2009, 56(4): 1261-1263.

[4] Ku C T, Hung K C, Wu T C, et al. Wavelet-based ECG data compression system with linear quality control scheme[J]. Biomedical Engineering, IEEE Transactions on, 2010, 57(6): 1399-1409.

[5] S. Lee, J. Kim, M. Lee, A real-time ECG data compression and transmission algorithm for an ehealth device, IEEE Trans. Biomed. Eng. 58 (9) (2011) 2448-2455.

[6] N.Rodrigues,E.daSilva,S.deFaria,V.daSilva,M.deCarvalho,etal.,ECGsignal compression based on DC equalization and complexity sorting, IEEE Trans. Biomed. Eng. 55 (7) (2008) 1923-1926.

[7] H.-H. Chou, Y.-J. Chen, Y.-C. Shiau, T. son Kuo, An effective and efficient compression algorithm for ECG signals with irregular periods, IEEE Trans. Biomed. Eng. 53 (6) (2006) 11981205. http://dx.doi.org/10.1109/TBME.2005.863961.

[8]A. Khalaj, H.M. Naimi, New efficient fractal based compression method for electrocardiogram signals, in: Canadian Conference on Electrical and Computer Engineering, CCECE'09, IEEE, 2009, pp. 983-986. 
[9]Ibaida A, Al-Shammary D, Khalil I. Cloud enabled fractal based ECG compression in wireless body sensor networks[J]. Future Generation Computer Systems, 2014, 35: 91-101.

[10]Al-Shammary D, Khalil I. Dynamic fractal clustering technique for soap web messages[C]//Services Computing (SCC), 2011 IEEE International Conference on. IEEE, 2011: 96103.

[11] The transform and data compression handbook[M]. CRC press, 2000. 